MATEC Web of Conferences 51, 02008 (2016)

DOI: $10.1051 /$ matecconf/20165102008

(C) Owned by the authors, published by EDP Sciences, 2016

\title{
Development of Electric Wheelchair with Input of Force Feedback Joystick
}

\author{
Keisuke SUGIHARA ${ }^{1}$, Shinsaku FUJIMOTO ${ }^{1, a}$ and Koji YOSHIDA ${ }^{1}$ \\ ${ }^{1}$ Okayama University of Science, Engineering Graduate Course, \\ 1-1 Ridaicho, Kita-ku, Okayama, JAPAN
}

\begin{abstract}
The purpose of this study is to realize the high function of an electric wheelchair with the consideration of the driving environment. The orientation sensor is used to comprehend the driving environment. The estimated method can be obtained unknown parameters of the driving environment by the orientation sensor. The developed electricwheelchair operates by the force-feedback joystick. When the marketed wheelchairs drive on the slope or the side-slope, the wheelchairs flow to the slope lower direction. In this case, the wheelchairs might go to roadway side in JAPAN, and such situations are very dangerous. The force-feedback joystick is developed to avoid dangerous situation. The force-feedback joystick is combined with the orientation sensor to the force feedback of joystick. The effectiveness of the force-feedback joystick is confirmed by comparison experiments with the control performance of the wheelchair without the force feedback. Therefore, this study is expected to be useful to social welfare in the future.
\end{abstract}

\section{Introduction}

This study focuses on an electric wheelchair which is a welfare apparatus for mobility support of a physical handicapped person. The purpose of this study is to develop the electric wheelchair which can reduce the burden of user and caregiver by the consideration of the driving environment as such the slope or the bad road.

The general operation methods of the electric wheelchair[1] are a hand-rim, joystick and the handle. This study discusses about an electric wheelchair with the joystick type. When the marketed electricwheelchairs drive on the slope or the side-slope, wheelchairs flow to the slope lower direction (unintentional direction). In this case, wheelchairs might go to the roadway side, and such situations are very dangerous. Thus, it is necessary to consider the driving environment for the electric wheelchair. Therefore several kinds of inertia sensors measure the dynamic slope-angles, while moving the wheelchair. In order to avoid dangerous situations, the proposed electric-wheelchair is equipped the force-feedback (hereafter FFB) joystick system[2][3].

Figure 1 shows the control method of the FFB joystick. On the Figure 1, the blue arrow is the direction of the desired movement, a red arrow is the direction of the force-feedback, and a blue dashed line is the straight direction. The pale joystick is the position of center (neutral position). The

\footnotetext{
${ }^{\mathrm{a}}$ Corresponding author : fuji@are.ous.ac.jp
} 
dark joysticks is the user's mistake operation. This study is based on the control concepts as shown in Figure 1. The user avoids the mistake operation and danger by FFB joystick, according to the direction of the slope and the acceleration (and velocity) of the wheelchair. It is dangerous to operate the joystick to the straight direction on the side-slope[3]. If the user operates it to the slope direction as shown in Figure 1(a) and (d), the wheelchair suddenly accelerates. In addition, if the user gives the quantity of excessive operation to the slope direction (See Fuigure 1 (b) and (c)), the wheelchair becomes the same situation. Therefore, the joystick suppresses the mistake operation by the force feedback (direction of red-arrow). Since the proposed FFB joystick should not suppress the desired movement for the user, the maximum magnitude of the force feedback is $3 \mathrm{~N}$. However, the maximum magnitude must be adjusted by each user.

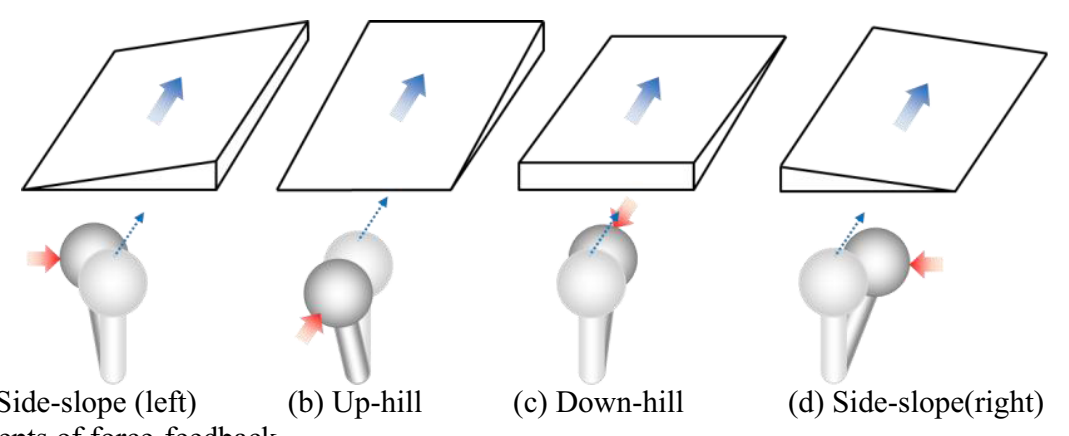

Figure 1. Concepts of force-feedback.

\section{Structure of FFB joystick[4]}

The FFB joystick is developed as the same excursion of both axes (See Figure 2). When wheelchair users release the hand from a joystick by the operating mistake, the linear spring is attached to return to an origin (neutral position) of each axis. In addition, DC motor and potentiometer are equipped to axes of $\mathrm{x}$-axis and $\mathrm{y}$-axis. Therefore, $\mathrm{x}$ and $\mathrm{y}$ positions of the joystick can be measured by two potentiometers.
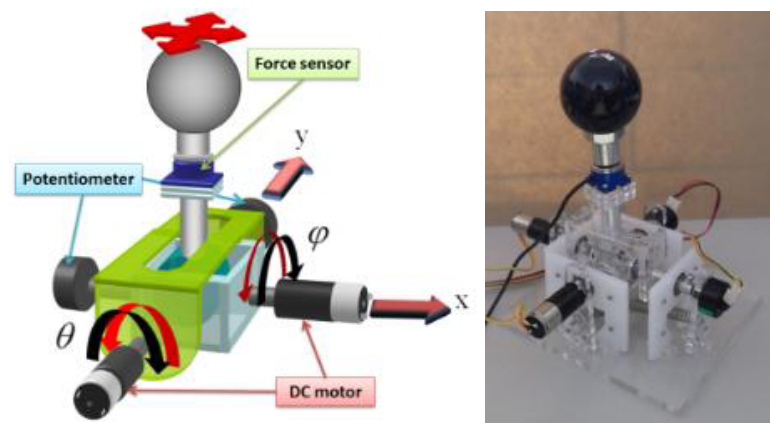

Figure 2. Structure FFB joystick and prototype of FFB joystick

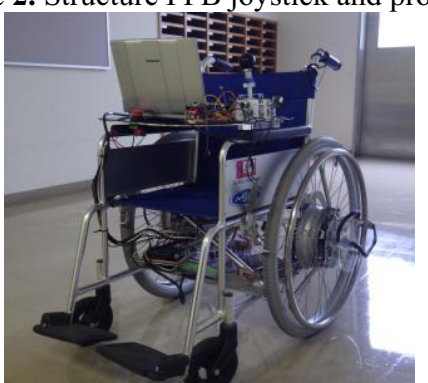

(a) Developed electric-wheelchair

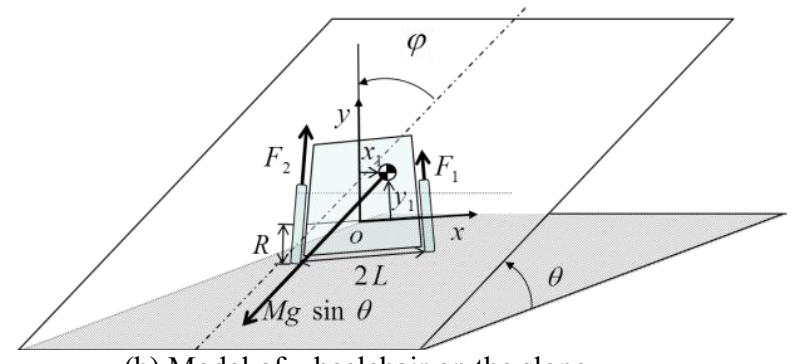

(b) Model of wheelchair on the slope

Figure 3. Developed electric-wheelchair and model of wheelchair on the slope 
Figure 3(a) shows the developed electric-wheelchair. When the marketed electric-wheelchair drives on the side-slope, the wheelchair goes to the slope direction against user's will. This case is caused by the difference(error vector) between the user's joystick operation(vector of desired movement) and the wheelchair's movement vector. Hereupon, the control error is modified by the FFB joystick in this study. In addition, the developed electric-wheelchair compensates the gravity term by slope angles as shown in Figure 3(b). Then, if the gravity compensation[5] is insufficient by changing mass of wheelchair, the FBB joystick is also used to compensate the modelling error.

The gravity terms of both wheels $\left(\mathrm{F}_{1}:\right.$ Right, $\mathrm{F}_{2}:$ Left $)$ are the following equations.

$$
\begin{gathered}
F_{1}=M g \sin \theta\left\{\sin \varphi-\frac{\left(L+y_{1}\right) \sin \varphi-x_{1} \cos \varphi}{2 L}\right\} \\
F_{2}=M g \sin \theta\left\{\frac{\left(L+y_{1}\right) \sin \varphi-x_{1} \cos \varphi}{2 L}\right\}
\end{gathered}
$$

where $M$ is the total mass of wheelchair, $g$ is acceleration of gravity, $R$ is wheel radius, $2 L$ is the distance between the axes, $\theta$ is angle of slope, $\varphi$ is direction angle of wheelchair and $x_{1}, y_{1}$ are positions of the center of gravity, respectively. The upper equation is the gravity compensation of each wheel.

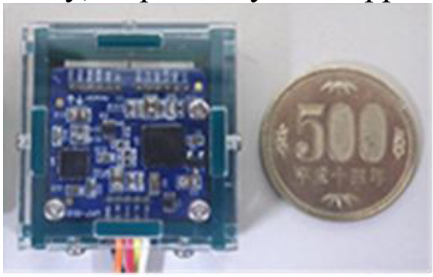

(a) Orientation sensor

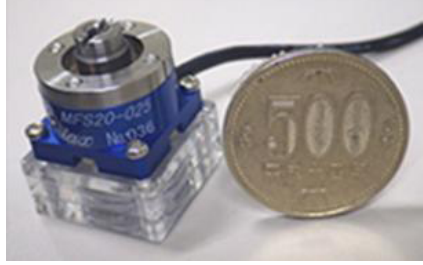

(b) 3-axis force sensor

Figure 4. Orientation sensor (Roll Pitch Yaw angle) and 3-axis force sensor for FFB joystick

This study adopted the orientation sensor (CH Robotics: UM7-LT orientation, size: $27 \times 26 \times 6.6 \mathrm{~mm}$, mass: $3.5 \mathrm{~g}$ ) to perform gravity compensation (See Figure 4(a)). This sensor can be measured the dynamic slope-angles $(\theta$ and $\varphi)$ in real time. The gravity compensation of equations (1) and (2) is calculated by using the sensor data.

In addition, Figure 1 and Figure 4(b) show the 3-axis force sensor for FFB joystick. 3-axis force sensor (Nippon Liniax Co. Ltd.: MFS20-025, size: $20 \times 20 \times 18 \mathrm{~mm}$, mass: $26 \mathrm{~g}$, Moment $M x, M y$ : $0.25 \mathrm{Nm}$, Force $F \mathrm{z}: 50 \mathrm{~N})$ is attached to the central part of the joystick in order to measure the operating moments (about $\mathrm{x}$ and $\mathrm{y}$ axes) and force (z-axis).

The sensor can be also measured the operating force of z-axis. In other words, this sensor is used to determine that the user is holding the joystick. In addition, if the wheelchair passes on the slope (or if the speed of wheelchair is too fast), FFB joystick presents the force-feedback to the user. And the proposed wheelchair is controlled by the joystick such that the user is not dangerously.

\section{Force-Feedback Method}

The positions ( $x$ and $y$ ) of the FFB joystick system can be expressed in following equation easily. Figure 5 shows the joystick coordinate system.

$$
\left\{\begin{array}{l}
x=L \cos \varphi \sin \theta=f_{1}(\boldsymbol{q}) \\
y=-L \sin \varphi \cos \theta=f_{2}(\boldsymbol{q}) \quad \because \boldsymbol{q}=\left[\begin{array}{ll}
\varphi & \theta
\end{array}\right]^{\mathrm{T}}
\end{array}\right.
$$

where $L$ is the length of the joystick, $\varphi$ and $\theta$ are rotary angles of x-axis and y-axis. 


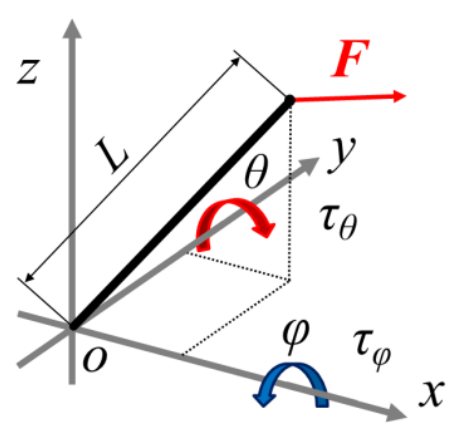

Figure 5. Joystick coordinate system.

The equation (3) is differentiated at time, and tip velocity of joystick is given by:

where $\boldsymbol{J}(\boldsymbol{q})$ is Jacobian matrix.

$$
\left[\begin{array}{c}
\dot{x} \\
\dot{y}
\end{array}\right]=\boldsymbol{J}(\boldsymbol{q}) \dot{\boldsymbol{q}}=\left[\begin{array}{ll}
-L \sin \varphi \sin \theta & L \cos \varphi \cos \theta \\
-L \cos \varphi \cos \theta & L \sin \varphi \sin \theta
\end{array}\right]\left[\begin{array}{c}
\dot{\varphi} \\
\dot{\theta}
\end{array}\right]
$$

In addition, linear spring is attached to the edge of each axis in order to return to neutral positions. Therefore dynamic models of the prototype joystick are given by:

$$
\left\{\begin{array}{l}
I_{\varphi} \ddot{\varphi}+D_{\varphi} \cdot \dot{\varphi}+K_{\varphi} \cdot \varphi=\tau_{M \varphi}-\tau_{\varphi} \\
I_{\theta} \ddot{\theta}+D_{\theta} \cdot \dot{\theta}+K_{\theta} \cdot \theta=\tau_{M \theta}-\tau_{\theta}
\end{array}\right.
$$

where $I_{*}$ are the inertia moments, $D_{*}$ are coefficients of viscous friction and $K *$ are spring constants, respectively. $(\because *=\varphi$ or $\theta)$

Assume that the operation of the joystick is in a static state, from principle of virtual work, the torques of motors can be expressed as:

$$
\left[\begin{array}{l}
\tau_{M \varphi} \\
\tau_{M \boldsymbol{\theta}}
\end{array}\right]=\left[\begin{array}{ll}
-L \sin \varphi \sin \theta & -L \cos \varphi \cos \theta \\
L \cos \varphi \cos \theta & L \sin \varphi \sin \theta
\end{array}\right]\left[\begin{array}{l}
f_{x} \\
f_{y}
\end{array}\right]+\boldsymbol{K}\left[\begin{array}{l}
\varphi \\
\theta
\end{array}\right]
$$

Hence, upper equation holds:

$$
\boldsymbol{\tau}_{M}=\boldsymbol{J}^{\mathrm{T}}(\boldsymbol{q}) \cdot \boldsymbol{F}+\boldsymbol{K} \cdot \boldsymbol{q} \quad \because \boldsymbol{K}=\operatorname{diag}\left(K_{\varphi} \quad K_{\theta}\right)
$$

If the external force $\boldsymbol{F}$ is given by the designer and angular vector $\boldsymbol{q}$ can be measured, we can derive $\boldsymbol{\tau}_{M}=\left[\tau_{M \varphi} \tau_{M \varphi \theta}\right]^{\mathrm{T}}$ (motor torques). Therefore, the FFB joystick can present the force feedback to the user. In addition, if the relation between the force sensor and motor torques can calibrate, note that the force-feedback with the expensive force sensor is not necessary for FFB joystick.

\section{Verification Experiment of Force-Feedback Method}

The verification experiment is performed to confirm the effectiveness of force-feedback method. The experimental method is as follows. When the user operated joystick to $\mathrm{x}$ and $\mathrm{y}$ axes, 3-axis force sensor is measured the feedback forces (Desired forces:2N) of the user. And, the user is presented the force by the FFB joystick.

However, 3-axis force sensor is used only the force sensing. In other words, the force sensor isn't necessary for the force-feedback.

Figure 6 shows the experimental results of the proposed method(Equation (7)), desired forces (2N) are shown to the user. This result is confirmed the effectiveness of proposed force-feedback method. 


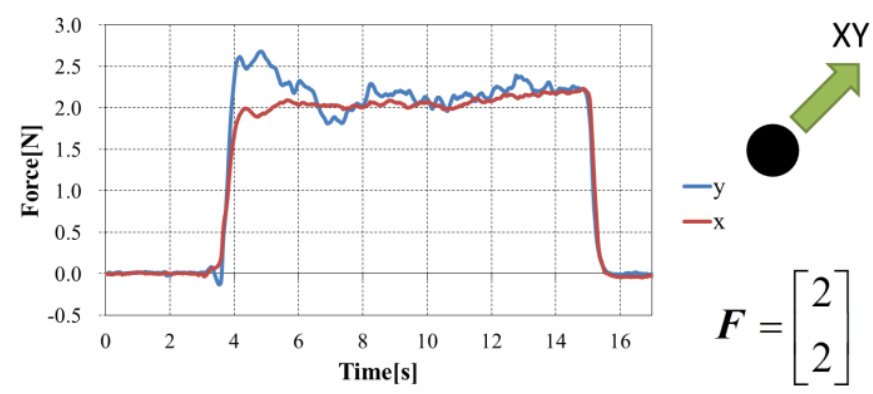

Figure 6. Experimental results of the Force-Feedback Method(Proposed method)

\section{Measurement of Orientation Sensor}

Figure 7 shows the result which the orientation sensor was measured on the side-slope. Red line is the direction of movement(Sagittal plane). Blue line is right and left direction(Lateral plane). These lines denote angles of inclination. The orientation sensor could measure the inclination angle of about 5 degrees in the case of the side-slope. From Figure 7, a little vibration occurred in direction of movement. The inclination angle(Red line) was measured the angular error within \pm 1 degree. However, the angle of the wheelchair oscillates actually in the direction of movement by means of the acceleration (and deceleration) of the wheelchair. The right and left direction was measured about 5 degrees. Therefore, the orientation sensor is able to be applied to an electric wheelchair system.
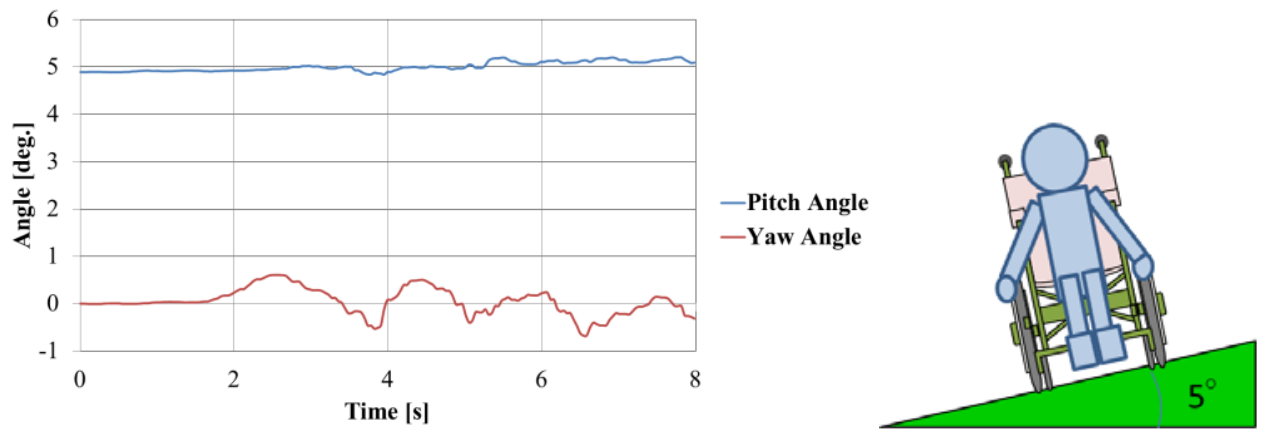

Figure 7. Measurement results of the side-slope(Slope angle: $\theta=5$ degrees)

\section{Driving Experiment}

This chapter is shown by the experimental results about the effectiveness of the FFB joystick. This experiment was performed on the side-slope of 2 degrees. First, the wheelchair without the forcefeedback was operated on the side-slope. Next, the wheelchair with the force-feedback was operated in same environment. The user generally operates the joystick in the forward direction. But, the user might modify the direction of the FFB joystick by the force-feedback.

The line shows the driving path of the wheelchair as shown in Figure 8. Figure 8(a) shows the driving path of the wheelchair that was equipped the normal joystick without FFB. Figure 8(a) shows that the wheelchair(Conventional wheelchair) greatly flowed to the slope direction(Left side). In case of this experiment, the user stopped the wheelchair at around 2 meters because the user was dangerous. Figure 8(b) shows the driving path of the wheelchair that equipped the FFB joystick. Since the behavior of the wheelchair is different from the direction vector of the joystick as soon as the wheelchair starts, the movement direction is modified by the force-feedback. Thus, a wheelchair with the FFB joystick didn't flow to the slope direction(Left side). When the FFB joystick was used, the position error was about $20 \mathrm{~cm}$. But, the position error of the conventional wheelchair was $60 \mathrm{~cm}$. Therefore, the effectiveness of the FFB joystick was confirmed by these experiments on the side-slope. 


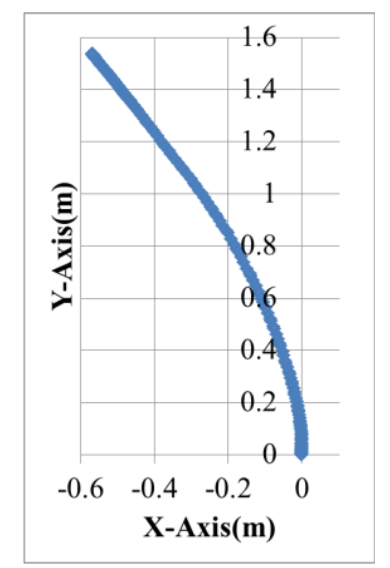

(a) Normal joystick without FFB

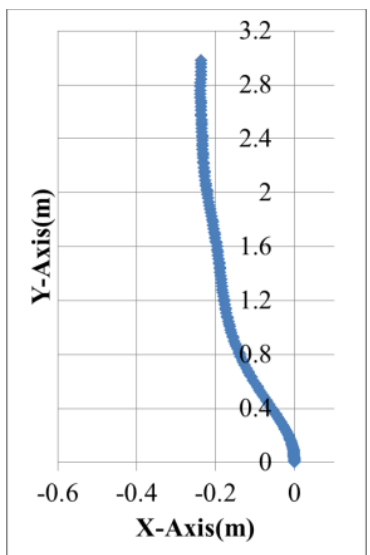

(b) FFB joystick

Figure 8. Comparison with the driving paths of the wheelchairs without FFB and with FFB

\section{Conclusions and Future Work}

This study focused on the electric wheelchair of the joystick input and the FFB joystick was produced. On the other hands, the sensor system was constructed to measure the driving environment.

As a result, the dynamic orientation could be measured when a target object (wheelchair) moved. The FFB joystick was produced and force-feedback method was proposed. In addition, the effectiveness of the FFB joystick was confirmed by experimental results on the side-slope.

In future work, this study will propose the method of force-feedback for the slope (up and down hill), an evaluation method of the joystick and an adaptive control design with consideration of the driving environment.

\section{Acknowledgements}

This work was supported by Grant-in-Aid for Scientific Research (C) 25350696. In addition, we are deeply grateful to Professor M. Kurisu (Tokyo Denki University) about that we could receive precious time and advice about software development the orientation sensor (UM-7).

\section{References}

[1] S. Watanabe, Development of Power Assisted Wheelchair Easy to Drive on Slope, University of Tokyo, Graduation thesis (2004) (in Japanese)

[2] V. Vashista, X. Chen and S. K. Agrawal, Adult-Human Learning on a Robotic Wheelchair Using a Force Feedback Joystick, Proceedings of the 1st International and 16th National Conference on Machines and Mechanisms (iNaCoMM2013), 794-799(2013)

[3] M.A.H. Abdelkader, G. Bourhis and B. Cherki, Haptic Feedback and Motor Disability: A Preliminary Study, HuMaN 07 proceedings, Timimoun(2007)

[4] Brubaker CE, McLaurin CA, McClay IS, Effects of side slope on wheelchair performance, Journal of Rehabilitation Research and Development, 23-2 , 55-57(1986)

[5] K. Sugihara, S. Fujimoto K. Yoshida, Development of Electric Wheelchair with Input of Force Feedback Joystick, Proceedings of the 24th Annual Conference of the SICE Chugoku Chapter, 9091 (2015) (in Japanese) 